\title{
The Effect of the Finite Ion Larmor Radius on the Kelvin-Helmholtz Instability
}

\section{Melchior, H.; Popovic, M.}

Publication date:

1967

Document Version

Publisher's PDF, also known as Version of record

Link back to DTU Orbit

Citation (APA):

Melchior, H., \& Popovic, M. (1967). The Effect of the Finite Ion Larmor Radius on the Kelvin-Helmholtz Instability. Risø National Laboratory. Denmark. Forskningscenter Risoe. Risoe-R No. 158

\section{General rights}

Copyright and moral rights for the publications made accessible in the public portal are retained by the authors and/or other copyright owners and it is a condition of accessing publications that users recognise and abide by the legal requirements associated with these rights.

- Users may download and print one copy of any publication from the public portal for the purpose of private study or research.

- You may not further distribute the material or use it for any profit-making activity or commercial gain

- You may freely distribute the URL identifying the publication in the public portal

If you believe that this document breaches copyright please contact us providing details, and we will remove access to the work immediately and investigate your claim 
The Effect of the Finite Ion Larmor Radius on the Kelvin-Helmholtz Instability

\author{
by \\ Henning Melchior and M. Popovic \\ The Danish Atomic Energy Commission \\ Research Establishment Risö \\ Physics Department
}

\begin{abstract}
Excitation of the Kelvin-Helmholtz instability has been observed in a Q-machine when a sufficiently large velocity shear exists between adjacent layers of the plasma column.

In an interpretation of this observation, possible effects of the finite ion Larmor radius and the collisional viscosity were neglected. The purpose of our study is to remove the first of these simplifications. With the finite ion Larmor radius included, the growth rate of the instability is computed for several values of the velocity shear and different $e$-folding lengths of the density profiles.
\end{abstract}




\section{Contents}

Page

1. Intr luction $\ldots \ldots \ldots \ldots \ldots \ldots \ldots \ldots \ldots \ldots \ldots \ldots \ldots, \ldots \ldots \ldots$

?. Assumptions asout the Plasma ..................... 3

3. Equations $\ldots \ldots \ldots \ldots \ldots \ldots \ldots \ldots \ldots \ldots \ldots \ldots \ldots \ldots \ldots \ldots \ldots \ldots \ldots$

4. First-order Perturbed State $\ldots \ldots \ldots \ldots \ldots \ldots \ldots \ldots \ldots \ldots, 5$

5. Computations and Results $\ldots \ldots \ldots \ldots \ldots \ldots \ldots \ldots \ldots, 8$

References $\ldots \ldots \ldots \ldots \ldots \ldots \ldots \ldots \ldots \ldots \ldots \ldots \ldots \ldots . \ldots \ldots$

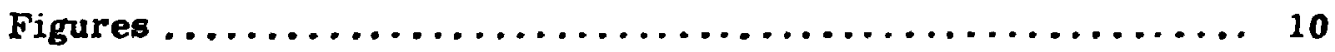




\section{Introduction}

The purpose of this paper is to determine the effect of the finite ion Larmor radius on the Kelvin-Helmholtz instability in a fully ionized plasma. The possible occurrence of the Kelvin-Helmholtz instability in a fully ionized plasma immersed in a magnetic field has been treated by $N$. D'Angelo ${ }^{1)}$ and later by $S$. v. Goeler ${ }^{2)}$. The effect of the finite Larmor radius was, however, not taken into account, and this paper describes an attempt to do so by the method indicated by K. V. Roberts and J. B. Taylor ${ }^{3)}$ and later used by F. i. . Chen ${ }^{4)}$.

\section{Assumptions about the Plasma}

The ratio between the particle pressure and the magnetic pressure is supposed to be extremely smail so that changes in the magnetic field due to the motion of the plasma can be neglected (low- $\beta$ approximation). In a Cartesiar frame of reference the magnetic field is taken to be oriented along the z-axis in the positive direction. A density gradient is supposed to exist aiong the $x$-axis, the unperturbed density distribution being of the form $n_{0}(x)=\bar{n}_{0} e^{-\lambda x}$. The ions stream along the magnetic lines with a velocity that is constant along each line, but different for different lines. This shear in velocity may give rise to the Kelvin-Helmholtz instability.

\section{Equations}

The particle motion is described by the two-fluid equations, in which the effects of the finite Larmor radius is included via the viscosity etreas tensor for the ions. The electron viscosity can be shown to be negligible.

The macroscopic equations $\mathrm{fcr}$ the ions are

$$
\begin{aligned}
& \frac{\partial n_{i}}{\partial t}+\nabla \cdot\left(n_{i-i}\right)=0 \\
& n_{i} m_{i}\left(\frac{\partial v_{i}}{i}+y_{i} \cdot \nabla y_{i}\right)=q n_{i}\left(v_{i} \times B-\nabla_{\phi}\right)-K T_{!} \nabla n_{i}-\nabla I .
\end{aligned}
$$

In addition we have an analogous get of equations for the electrons, in which, however, the viscosity term is neglected. In the colligionless limit the terme of the viscosity stress tensor are 


$$
\begin{aligned}
& -\Pi_{x x}=\Pi_{y y}=\frac{n_{i} K T_{i}}{\omega_{c i}} U_{x y} \\
& \Pi_{z z}=0 \\
& -\Pi_{x y}=-\Pi_{y x}=\frac{1}{2} \frac{n_{i} K T_{i}}{\omega_{c i}}\left(U_{y y}-U_{x x}\right) \\
& -\Pi_{x z}=-\Pi_{z x}=2 \frac{n_{i} K T_{i}}{\omega_{c i}} U_{y z} \\
& -\Pi_{y z}=-\Pi_{z y}=2 \frac{n_{i} K T_{i}}{\omega_{c i}} U_{x z}
\end{aligned}
$$

$U_{i j}$ are the components of the divergenceless symmetrical velocity gradient tensor, often called the rate-of-shear tensor. The components are given by

$$
\begin{aligned}
& u_{x x}=\frac{1}{3}\left(2 \frac{\partial v_{i o x}}{\partial x}-\frac{\partial v_{i o y}}{\partial y}-\frac{\partial v_{i o z}}{\partial z}\right) \\
& u_{y y}=\frac{1}{3}\left(2 \frac{\partial v_{i o y}}{\partial y}-\frac{\partial v_{i o x}}{\partial x}-\frac{\partial v_{i o z}}{\partial z}\right) \\
& u_{x y}=\frac{1}{2}\left(\frac{\partial v_{i o y}}{\partial x}+\frac{\partial v_{i o x}}{\partial y}\right) \\
& u_{x z}=\frac{1}{2}\left(\frac{\partial v_{i o z}}{\partial x}+\frac{\partial v_{i o x}}{\partial z}\right) \\
& u_{y z}=\frac{1}{2}\left(\frac{\partial v_{i o z}}{\partial y}+\frac{\partial v_{i o y}}{\partial z}\right) .
\end{aligned}
$$

When we consider a steady equilibrium solution of the type

$$
v_{\text {iox }}=0, \quad v_{i o y}=\text { const. }, \quad v_{i o z}=v_{i o z}(x)
$$

with a constant, or varishing zern-order electric field in the $x$ direction, the continuity equation and the $y$ and $z$ components of the momentum equation are automatically satisfied. The $x$-component of the momentum equation imposes the following conditions for the pressure gradient drift velocity $v_{i o y}$ 


$$
v_{\text {ioy }}=-\frac{c_{i}^{2}}{\omega_{c_{i}}} \lambda:
$$

where

$$
c_{i}^{2}=\frac{K T_{i}}{m_{i}} \quad \text { and } \quad c_{c_{i}}=\frac{q B}{m_{i}} \text {. }
$$

\section{First-orcer Perturbed State}

Stability conditions are obtained by analysing the perturbed, linearized macroscopic equations. In order to do so, we pertirb the various quantities from the zero-order state by writing

$$
n_{i}=n_{i 0}+n_{i 1}, \quad v_{i}=v_{i 0}+v_{i 1}, \quad \varphi=\varphi_{0}+\varphi_{1},
$$

where

$$
n_{\text {il }} \ll<n_{\text {io }} \text {, etc. }
$$

With these quantities in the governing macroscopic equations, and with higher-order terms neglected, one obtains after subtracting the zero-order equations:

$$
\begin{aligned}
& \frac{\partial n_{i 1}}{\partial t}+n_{i 0} v \cdot y_{i 1}+y_{i 1} \cdot \nabla_{n_{i 0}}+v_{i 0} \cdot \nabla_{n_{i 1}}=0 \\
& n_{i o} m_{i} \frac{\partial v_{i 1}}{\partial t}+m_{i} n_{i o} v_{i l} \cdot \nabla v_{i o}+m_{i} n_{i o} v_{i o} \cdot \nabla v_{i 1}+K T \nabla n_{i 1}+
\end{aligned}
$$

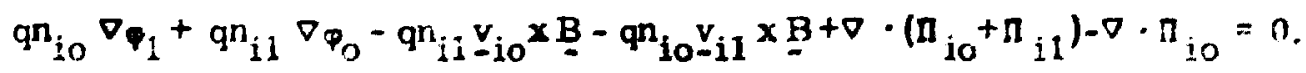

We assume the solutions for small-amplitude perturbations to be $n$ ? the form

$$
\begin{aligned}
& n_{i 1}=\dot{n}_{i 1} f(x) e^{i\left(k_{y} y+k_{z} z-\omega t\right)} \\
& v_{i 1}=v_{11}(x) e^{i\left(k_{y} y+k_{z} z-\omega t\right)} \\
& \nabla_{1}=\nabla_{1}(x) e^{i\left(k_{y} y+k_{z} z-\omega t\right)} .
\end{aligned}
$$


With a zero-order density distribution

$$
n_{i 0}(x)=\bar{n}_{i 0} e^{-\lambda x}
$$

we have

$$
\frac{\partial}{\partial x}\left(\frac{n_{i 1}}{n_{i 0}}\right)=\frac{1}{n_{i 0}} \frac{\partial l_{1}}{\partial x}+\lambda \frac{n_{i 1}}{n_{i 0}} .
$$

By assuming that the velocities are weak functions of $x$, and by making use of the equations (3), (4), (5), (8), (9), and (10), we can transform equations (6) and (7) to

$$
\begin{aligned}
& -i \Omega \frac{n_{i l}}{n_{i 0}}+i k_{y} v_{i l y}+i k_{z} v_{i l z}-\lambda v_{i l x}=0 \\
& -i \Omega v_{i 1 x}-\omega_{c i} v_{i 1 y}+c_{i}^{2} \frac{\partial}{\partial x} \frac{n_{i 1}}{n_{i 0}}+\frac{g}{m_{i}} \frac{\partial p l}{\partial x}= \\
& \frac{c_{i}^{2}}{w_{c i}}\left[-\frac{i}{2} \lambda k_{y} v_{i 1 x}-\frac{1}{2} k_{y}{ }^{2} v_{i l y}-k_{y} k_{z} v_{i 1 z}-k_{z}^{2} v_{i 1 y}\right]: \\
& -i g V_{i 1 y}+i k_{y} c_{i}^{2} \frac{n_{i 1}}{n_{i 0}}+i k_{y} \frac{q}{m_{i}} \Phi_{1}+\omega_{c i} v_{i 1 x}=\frac{c_{i}^{2}}{w_{c i}}-\frac{i}{2} \lambda k_{y} v_{i l y}+ \\
& \frac{1}{2} k_{y}^{2} v_{i 1 x}+k_{z}^{2} v_{i 1 x}+i k_{z} \frac{n_{i 1}}{n_{i 0}} \frac{\partial v_{i o z}}{\partial x} \\
& -i \propto v_{i 1 z}+\frac{\partial v_{i 0 z}}{\partial x} v_{i 1 x}+i k_{z} c_{i}^{2} \frac{n_{i 1}}{n_{i o}}+i k_{z} \frac{q_{11}}{m_{i}}=\frac{c_{i}^{2}}{\omega_{c i}}\left[-i \lambda k_{y} v_{i 1 z}\right. \\
& \left.-i \lambda k_{z} v_{11 y}+k_{y} k_{z} v_{i 1 x}+i k_{y} \frac{n_{i 1}}{n_{i 0}} \frac{\partial v_{i O z}}{\partial x}\right] \text { : }
\end{aligned}
$$

where $0 \omega-k_{y} v_{\text {ioy }}-k_{z} v_{i o z}$. 
All terms on the right-hand side of the momentum equations come from the anisotropic pressure tensor and are due to the effect of the finite Larmor radius.

This system of four equations with five unknowns is completed by the $z$-component of the momentum equation for electrons. To ottain this equation, we will change $q$ into $-q, \omega_{c i}$ into $-\omega_{c e}, c_{i}^{2}$ into $c_{e}^{2}$, and $n_{i}$ into $n_{e}$ in the corresponding ion equation. The ierms which account for the finite Larmor radius effect are neglected. Since charge neutrality is preserved, this equation, in the limit $\frac{m_{e}}{m_{i}} \rightarrow 0$ and for equal temperatures $T_{i}=T_{e}$, is
reduced to

$$
c_{i}^{2} \frac{\bar{n}_{i 1} f(x)}{\bar{n}_{j o} e^{-\lambda x}}=\frac{q q_{1}}{m_{i}}
$$

This gives the relation between perturbed density and potential. If the perturbation in the potential does not depend on $x$, the ratio $n_{1} / n_{0}$ will be constant, i. e. $f(x) \equiv e^{-\lambda x}$.

The following dimensionless quantities were introduced for convenience:

$$
\begin{aligned}
\Lambda & =p_{i}\left(\phi_{i}=\frac{c_{i}}{\omega_{c i}}\right) \\
a & =k_{y} \rho_{i} \quad i=k_{z} p_{i} \\
\downarrow & =\frac{\omega-k_{y} v_{\text {ioy }}-k_{z} v_{i o z}}{\omega_{c}} \\
v^{*} & =\frac{v}{c_{i}} \quad \frac{k_{i j}}{\lambda_{p i}}=\frac{b}{a} \\
k & =\frac{1}{\omega_{c i}} \frac{\partial v_{i o z}}{\partial x}
\end{aligned}
$$

Here is the frequency in the frame of the ions measured in units of ior cyclotron frequency while $A$ is the non-dimensional shear in velocity.

Converting equations (11) into a non-dimensional form and eliminating the perturbation in the potential throug: (12), we obtain 


$$
\begin{aligned}
& -\Delta V_{11 x}^{*}+i a V_{i 1 y}+i b V_{i 1 z}^{*}-i \phi \frac{\bar{n}_{i 1}}{\bar{n}_{i 0}}=0 \\
& \left(i \frac{a \Delta}{2}-i \phi_{i}\right) V_{i 1 x}^{*}+\left(\frac{a^{2}}{a}+b^{2}-1\right) V_{i 1 y^{*}}+a b V_{i 1 z}^{*}=0 \\
& \left(1-\frac{a^{2}}{2}-b^{2}\right) V_{i 1 x^{*}}+\left(i \frac{a \Delta}{2}-i \psi_{i}\right) V_{i 1 y^{*}}+(i b A+2 i a) \frac{\bar{n}_{i 1}}{\bar{i}_{i 0}}=0 \\
& (A-a b) V_{i 1 x}^{*}+i b \Delta V_{i 1 y}^{*}+\left(i a \Delta-i \phi_{i}\right) V_{i 1 z}^{*}+(2 i b+i a A) \frac{\bar{n}_{i 1}}{\bar{r}_{i 0}}=0 .
\end{aligned}
$$

The condition that the determinant of this set of equations vanish gives the dispersion relation. By some algebraic transformations the determinant is brought into a form where the fourth-order algebraic equation becomes equivalent to an eigenvalue problem for the magnitude D $=\left(\omega-k_{y} v_{\text {ioy }}-k_{z} v_{\text {ioz }}\right) / \omega_{c i}$

\section{Computations and Results}

The eigenvalue problem has been solved numerically on the IEM 7090 computer at the North European University Cumputing Center at Lyngby. In these computations the complex matrix was transformed to upper Hessenberg form by a modified version of the algorithm given by Dennis J. Mueller ${ }^{5}$ ) connected with the QR-transformation procedure given by Axel Ruhe ${ }^{6)}$.

For each of the values of $A$ (the non-dimensional shear in velocity), which was varied in 20 steps from 0 to 0.40 , the dispersion relation was solved for 20 different values of $k$, the numerical value of the propagation vector. $k$ was varied from an initial value of $10^{-4}$ to 1 in the following way:

$$
k_{n}=k_{n-1} \exp \left(\frac{1}{5} \ln 10\right) .
$$

This expression connects the actual value $k_{n}$ with the value $k_{n-1}$ used in the previous step. For a specific value of $\mathrm{A}, 20$ different solutions of the dispersion relation are obtained (corresponding to the different values of $k$ ). Out of these solutions the one with the largest positive value of the imaginary part of the frequency is chosen since it will be the most unstable one.

In fig. 1 the values of the imaginary part of the frequency are plotted against $A$, the non-dimersional shear. In these calculations, $\lambda c_{i} / \omega_{c i}=0.09$. Curves for different values of $a=k_{y} c_{j} / \omega_{c i}$ are shown. 
It is seen that the growth laze 0 ihe instability increases as a is varied from 0.084 to a maximum of abul: 0.75 . After this point, the growth rate decreases for a furtier increase in a. It may also be seen that in the case of large growth rates the disei the instability occurs for small values of the shear A (see fig. 3).

The curves in fig. 2 show the imaginary part of the frequency for different values of $\mathrm{A}$. It is seen tha: an increase in $\mathrm{A}$ has a stabilizing effect. In fig. 3 a comparison is presented between results of calculations performed with and without the finite l..rmor radius terms. In general, these terms will have a stabilizing effect, but as curve II shows, there may be unstable solutions with the finite Larmor radius taken into account where calculations without it give no instability at all.

In fig. 4 the dependence of the real part of the frequency on the magnitude of the propagation vector is shown. It is seen that the freouency increases with increasing $\mathrm{k}_{\mathrm{y}} \mathrm{c}_{\mathrm{i}} / \omega_{\mathrm{ci}}$, and that it is higher with the finite Larmor radius taken into account than without.

It is our intention later to verify experimentally the above results on the Q-machine at Riso. The results obtained in the present calculations support what has been found earlier by $N . D^{\prime} A^{A n g e l o}{ }^{1)}$ and $S . v$. Goeler ${ }^{2}$. So it is not imperative to include the finite Larmor radius effect in the description of the Kelvin-Helmholtz instability experiment in a Q-machine. We feel, however, that through these calculations one obtains a more accurate description of the Kelvin-Helmholtz instability in a fully ionized plasma.

\section{References}

1) N. D'Angeio, Phys, Fluids 9 , 1748 (1965).

2) S. v. Goeler, Phys. F'luids 9 , 818 (1966).

3) K. V. Roberts and J. B. Taylor, Phys. Rev. Letters E, 197 (1962).

4) Francis F. Chen, Phys. Fluids $\underline{3}, 1323$ (1965).

5) D. J. Mueller, Num. Nath. 3, 72 (1966).

6) Axel Ruhe, B1T 6 , 350 (1966). 


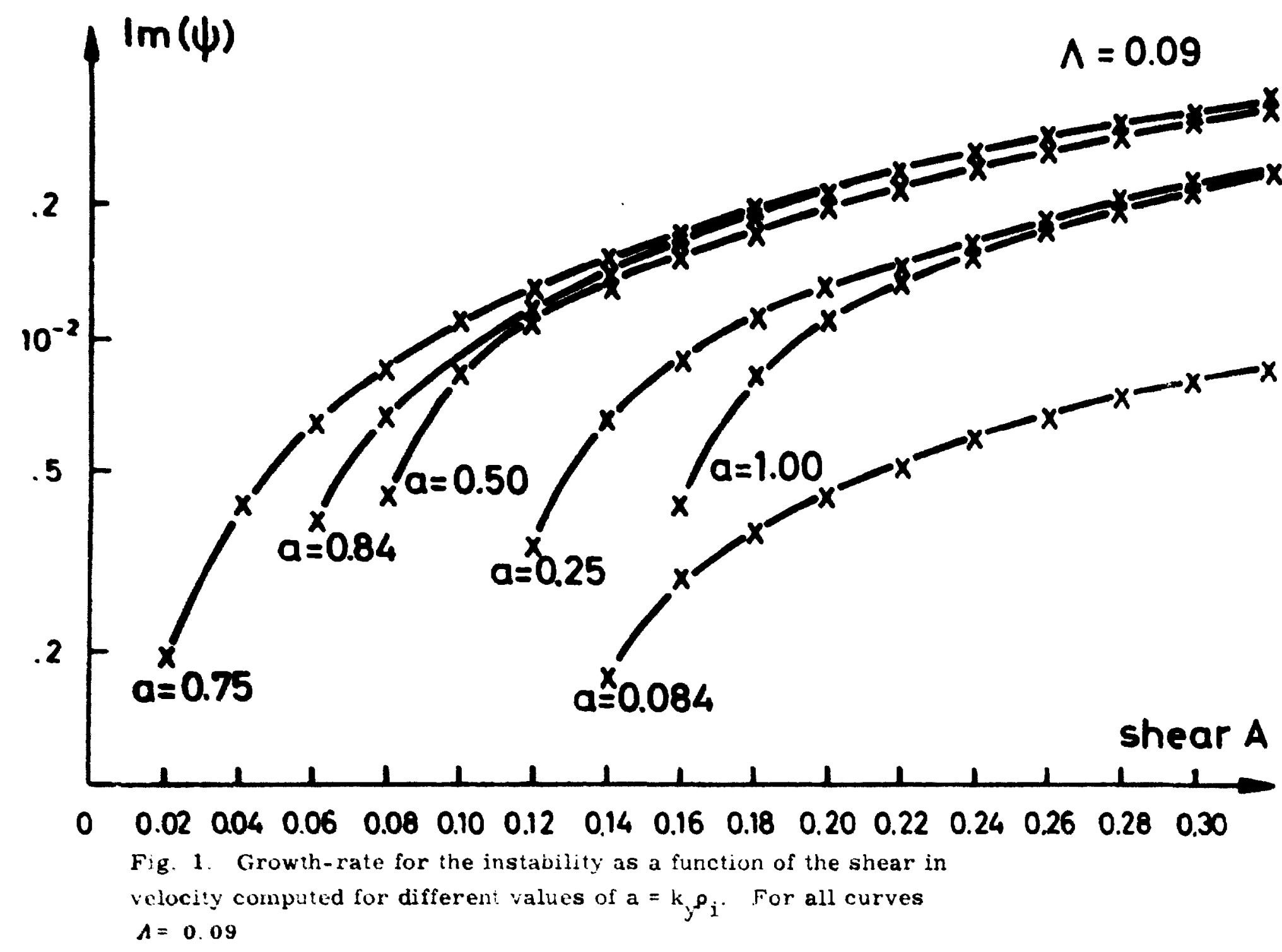




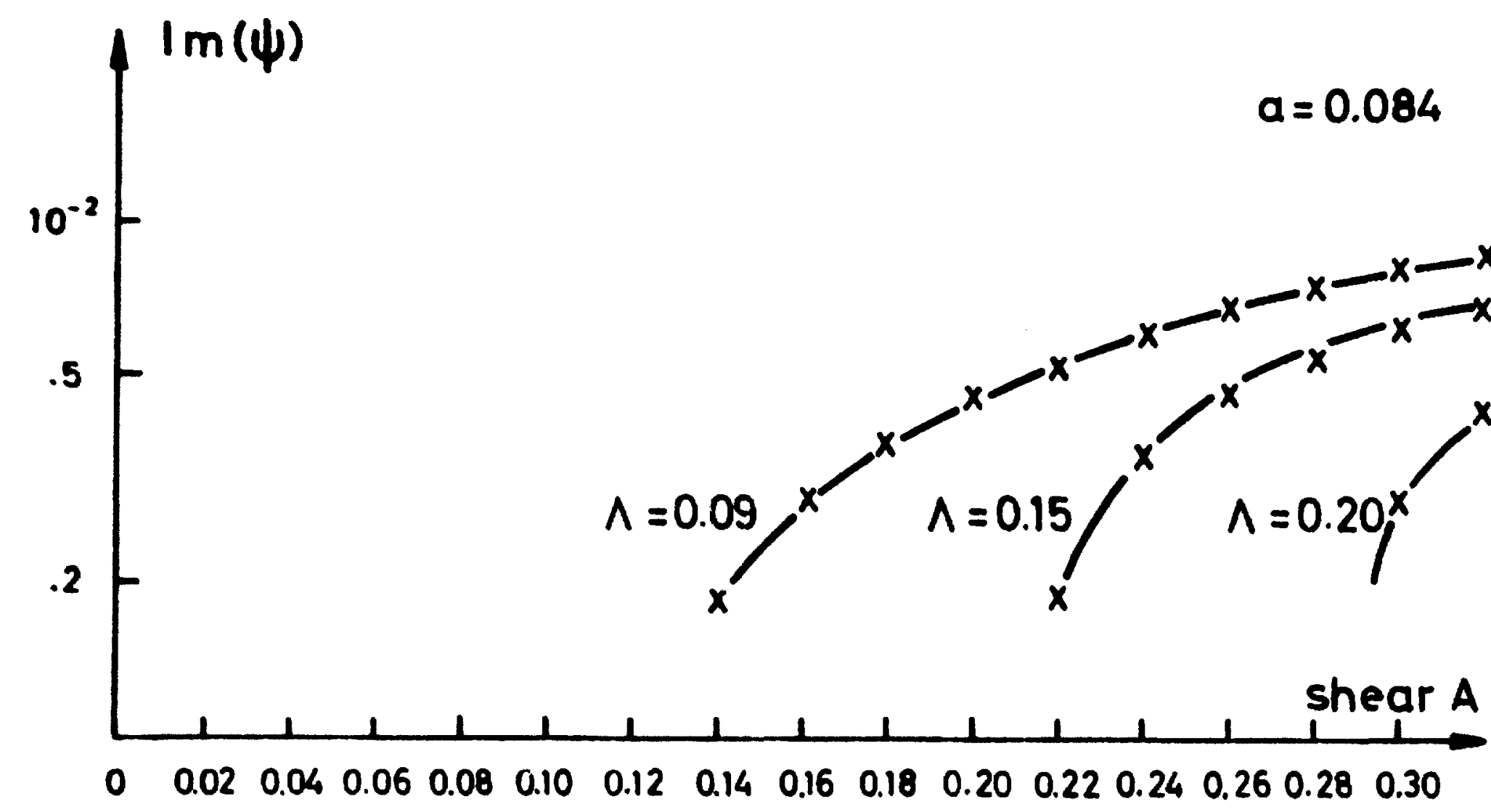

Fig. 2, Growth-rate for the instability as a function of the shear in

velocil computed for different values of $\Lambda=\lambda_{i}$. For all curves

$a=0.084$ 

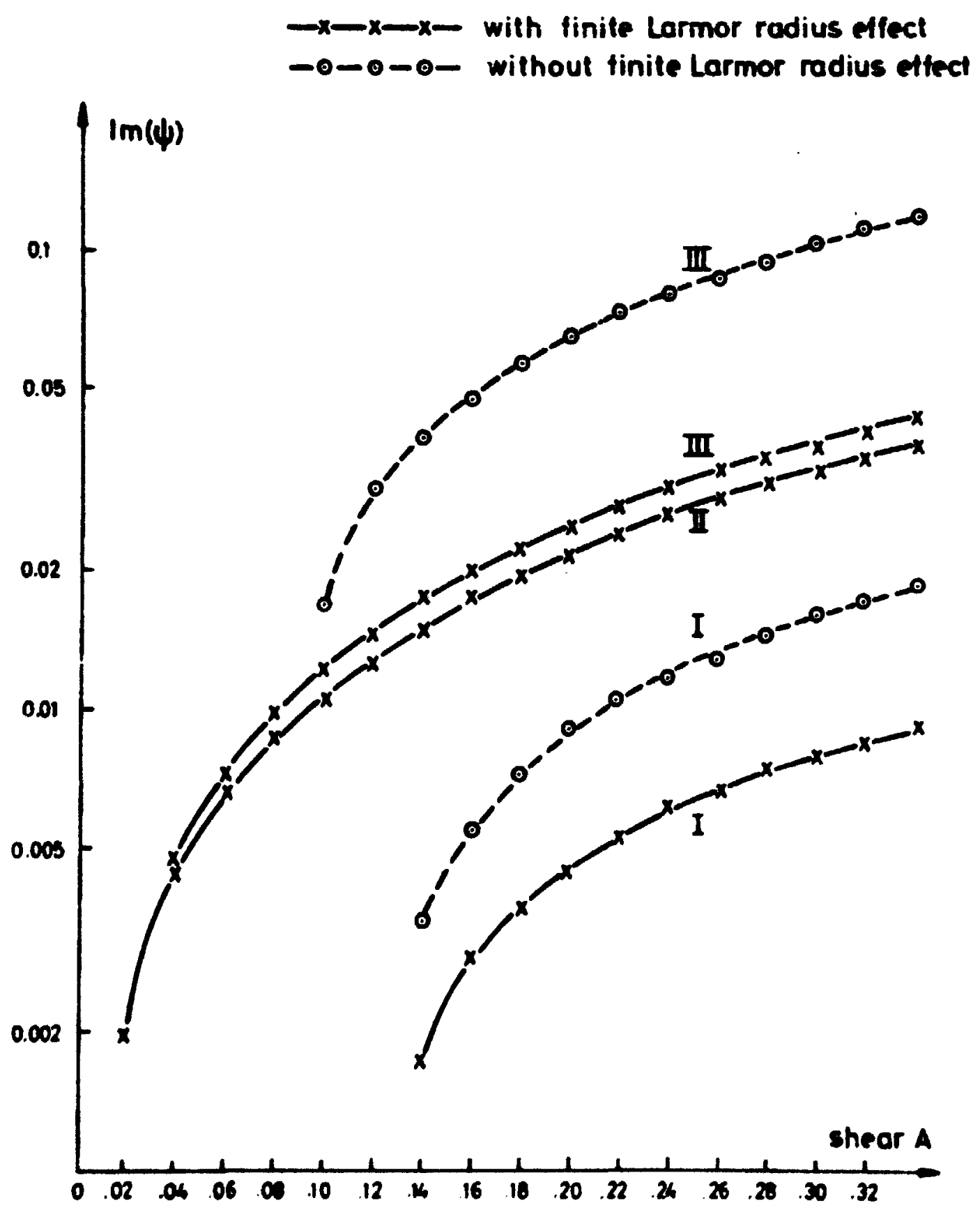

Fig. 3. The curves show the growth-rate of the instability for different values of $a=k_{y} \rho_{i}$ and $\Lambda=\lambda p_{i}$ computed with and without taking the finite Larmor radius effect into account. For the curves $1 \mathrm{a}=0.084$ and $\Delta=0.09$. For curve II $a=0.084$ and $\Delta=0.5$. For the curves Lit $a=0.84$ and $\Lambda=009$ 


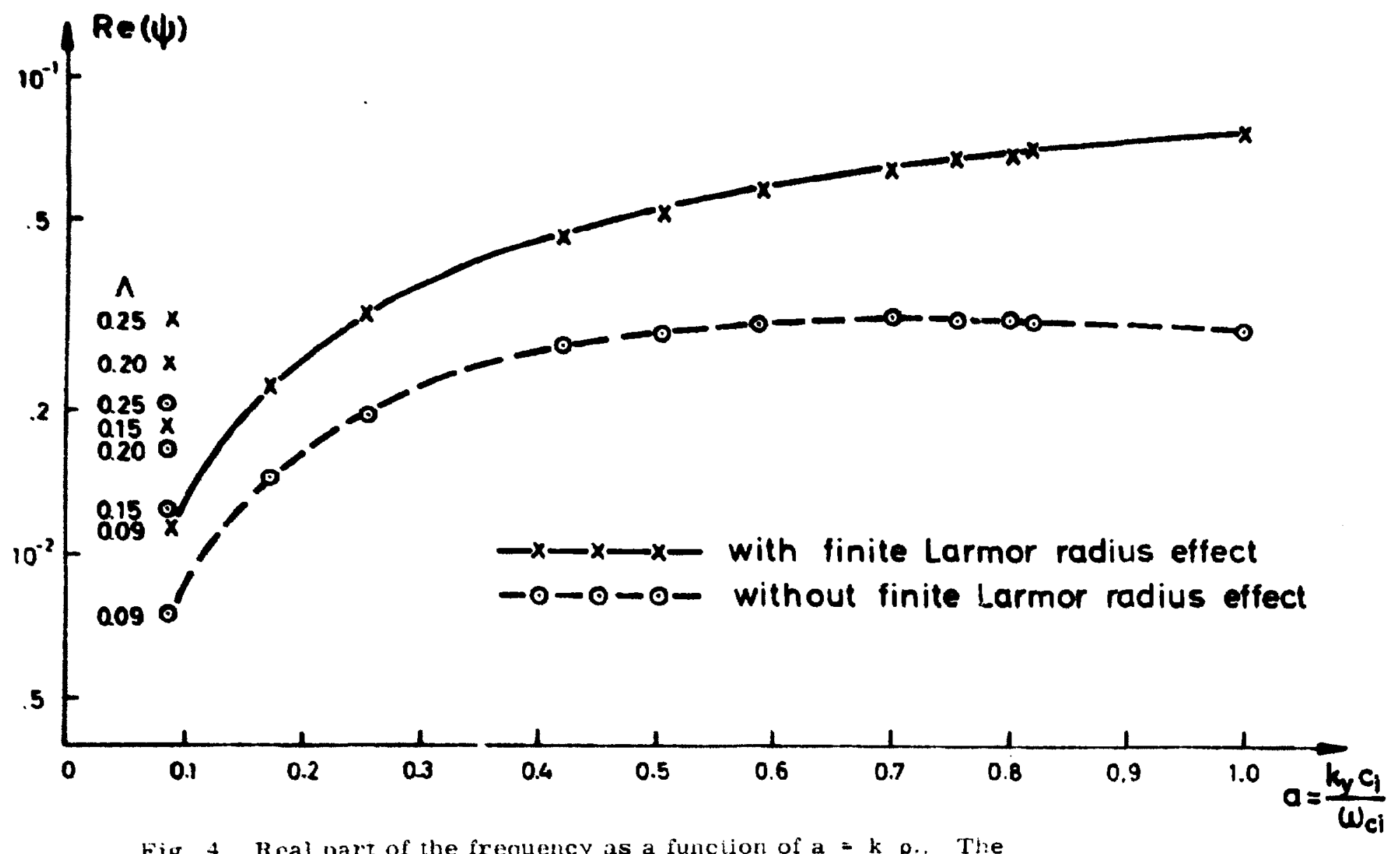

Fig. 4. Real part of the irequency as a funciion of $a=k \rho_{j}$. The curves for $\Lambda=0.09$ are computed with and without finite Larmor radius effect. 